\title{
Frequency and Course of Mild Cognitive Impairment in a Multiethnic Community
}

\author{
Jennifer J. Manly, PhD, ${ }^{1-3}$ Ming-X. Tang, PhD,,${ }^{1,4}$ Nicole Schupf, PhD, ${ }^{1,2,5,6}$ Yaakov Stern, PhD, ${ }^{1-3,6}$
} Jean-Paul G. Vonsattel, MD, ${ }^{2,7}$ and Richard Mayeux, MD, MSc ${ }^{1-3,5,6}$

\begin{abstract}
Objective: To examine incidence rates and antecedents of mild cognitive impairment (MCI) and Alzheimer's disease (AD) among diverse elders without dementia at the initial visit, and to examine the characteristics of elders with MCI who reverted to normal on follow-up examination.

Methods: A total of 2,364 Caribbean Hispanic, black, or non-Hispanic white subjects, aged 65 or older, who were free of dementia at initial evaluation were followed up every 18 to 24 months. Incidence rate of MCI and AD was determined by examination of neurological, medical, psychiatric, and neuropsychological function.

Results: Over 10,517 person-years, 21\% of normal elderly subjects progressed to MCI (annual incidence rate, 5.1\%; 95\% confidence interval, $4.6-5.6 \%$ ). Of those with MCI initially, 21.8\% were subsequently diagnosed with $\mathrm{AD}$ (annual incidence rate, $5.4 \%$; 95\% confidence interval, $4.7-6.3 \%$ ), $47 \%$ remained unchanged, and 31\% reverted to normal. Those with MCI were 2.8 times more likely to experience development of $\mathrm{AD}$ than normal elderly subjects. $\mathrm{MCI}$ with impairment in memory and at least one other cognitive domain was associated with greatest risk for progression to $\mathrm{AD}$ and was also least likely to revert to normal at follow-up. Consistent diagnosis of $\mathrm{MCI}$ or incident probable or possible $\mathrm{AD}$ was $60 \%$ sensitive and $94 \%$ specific for the pathological diagnosis of $\mathrm{AD}$.

Interpretation: Impaired memory and language were useful predictors of transition to AD. Reversion to normal from MCI was frequent, but those with impairment in more than one cognitive domain were more likely to progress or remain impaired than those with single-domain impairment. Clinical diagnosis of MCI does not always predict $\mathrm{AD}$ neuropathology.
\end{abstract}

Ann Neurol 2008;63:494-506

The term mild cognitive impairment (MCI) describes the transitional state between normal aging and Alzheimer's disease $(\mathrm{AD})^{1-3}$ or dementia. ${ }^{4}$ Implementation of the criteria for $\mathrm{MCI}$, as well as determination of the rates of progression from MCI to $\mathrm{AD}$ or dementia, has been an area of great interest, in part because identifying the earliest signs of dementia will be crucial for interventions to prevent or slow progression of decline in $\mathrm{AD}$ and for research on $\mathrm{AD}$ and other dementias. ${ }^{5}$

The prevalence of MCI, as well as progression rates to $\mathrm{AD}$ or dementia, vary depending on multiple factors such as implementation of MCI criteria, recruitment source, age at the initial assessment, and length of follow-up. Annual progression from MCI to dementia ranges from 12 to $17 \%^{1,3,6}$ in clinic-based studies, whereas lower progression rates have been observed in population- or community-based studies $\left(4-15 \%^{7-13}\right.$ per year). Furthermore, in population-based studies, there appears to be more frequent occurrence of "reversion to normal" among elderly people with
MCI than in clinical cohorts, ranging from 14 to $40 \% .^{8,9,11,13,14}$

In addition to the type of cohort, the other key methodological factors differing across studies of $\mathrm{MCI}$ include: (1) whether MCI diagnoses are assigned on a case-by-case basis in a consensus conference of expert clinicians or assigned purely objectively using neuropsychological, functional, and medical data; (2) whether the diagnoses were made based on data collected before formal criteria for MCI were published, and thus may not be entirely suitable for the application of Peterson criteria for MCI; (3) the extent to which nondemented older subjects with memory deficits are distinguished from those with cognitive deficits in nonmemory domains; (4) the extent to which those with isolated deficits in one cognitive domain are distinguished from those with impairment in multiple cognitive domains; (5) the test score "cutoff" (and thus the extent of impairment) used to define cognitive impairment; (6) the use of norms that adjust for age and
From the ${ }^{1}$ Gertrude H. Sergievsky Center; ${ }^{2}$ Taub Institute for Research on Alzheimer's Disease and the Aging Brain; ${ }^{3}$ Department of Neurology; Departments of ${ }^{4}$ Biostatistics and ${ }^{5}$ Epidemiology, School of Public Health; and Departments of ${ }^{6}$ Psychiatry and ${ }^{7} \mathrm{~Pa}-$ thology, Columbia University Medical Center, New York, NY.

Received Mar 16, 2007, and in revised form Oct 31. Accepted for publication Nov 27, 2007.
Published online Feb 25, 2008, in Wiley InterScience (www.interscience.wiley.com). DOI: 10.1002/ana.21326

Address correspondence to Dr Mayeux, Taub Institute for Research on Alzheimer's Disease and the Aging Brain, Columbia University Medical Center, 630 West 168th Street, P\&S Box 16, New York, NY 10032. E-mail: rpm2@columbia.edu 
other background factors such as years of school, sex, and race/ethnicity; (7) the extent to which subjective memory complaints are considered as a requirement for the diagnosis of MCI; (8) whether intact functional capacity was required for a diagnosis of $\mathrm{MCI}$; and finally, (9) whether follow-up diagnosis is made with the knowledge of prior diagnostic status.

Few longitudinal studies of MCI have been conducted among elderly people from diverse racial or ethnic groups ${ }^{14}$ or from those from other linguistic or educational ${ }^{8}$ backgrounds. Progression rates to $A D$ may differ among ethnically and educationally diverse elderly individuals with MCI because the cognitive tests used to classify "objective" cognitive impairment have poor specificity in these groups. ${ }^{15,16}$ Furthermore, compared with non-Hispanic white individuals, black and Hispanic individuals are more likely to have hypertension and diabetes, ${ }^{17,18}$ and the impact of these conditions on the incidence rates of MCI and on the progression to dementia from MCI is unknown.

In this study, we determined the incidence rates of MCI within a large population-based cohort of ethnically, linguistically, and educationally diverse elders without dementia or cognitive impairment at the beginning of the study. We also compared the incidence rates of $\mathrm{AD}$ and dementia among elders with or without MCI at baseline, and compared rates of progression across MCI subtypes and determined the antecedents for progression. We also examined the proportion and characteristics of elderly subjects with MCI who reverted to normal on follow-up examination and compared these data with subjects who still had MCI or progressed to $\mathrm{AD}$ at follow-up. Among a small subsample of elders who died and donated brain tissue, we performed a preliminary exploration of the relation of MCI classification to presence of $\mathrm{AD}$ neuropathology and final neuropathological diagnoses.

\section{Subjects and Methods}

The Columbia University Institutional Review Board approved this project. All individuals discussed the study with a trained research assistant and provided written informed consent before their baseline visit.

\section{Sampling Plan and Participants}

Participants were Medicare recipients aged 65 or older residing in three contiguous census tracts in Northern Manhattan, New York, in the neighborhoods of Washington/Hamilton Heights and Inwood who were asked to participate in a longitudinal study of aging, cognitive function, and dementia. The population from which participants were drawn was composed of individuals from several countries of origin representing three broadly defined ethnic categories (ie, Caribbean Hispanic, black, and non-Hispanic white of European ancestry). Participants were excluded if they did not speak English or Spanish. The study combined longitudinal data from two recruitment efforts in this community, one begin- ning in 1992 and the other in 1999. The sampling strategies and recruitment outcomes of these two cohorts are detailed in prior publications. ${ }^{19-21}$ Reevaluations occurred during follow-up waves that were spaced approximately 18 to 30 months apart. Beginning in 2002, participants in both cohorts were asked whether they were interested in brain donation.

As shown in Figure 1, combining the 1992 and 1999 cohorts resulted in a group of 4,308 potential participants for this study. Data were used from only those participants who had sufficient neuropsychological, functional, medical, and neurological information to determine the presence or absence of MCI using published criteria. ${ }^{19}$ We found that $3830(89 \%)$ participants had sufficient data. We computed the incidence rates for MCI and dementia in a sample excluding 615 individuals with prevalent dementia at the initial visit and 785 elders without longitudinal data. Among the 785 subjects without longitudinal data, 38\% refused further visits, $24 \%$ died, $20 \%$ could not be located, $11 \%$ moved out of the area, $6 \%$ could not be scheduled for a visit, and $1 \%$ were lost for some other reason. Therefore, the "overall" rate of follow-up in this sample was $76 \%$ including deaths. The follow-up rate among people who were alive at the time of follow-up was $81 \%$. For these analyses, we further excluded $66(2.7 \%)$ of the 2,430 participants who did not have dementia at first visit and were seen for at least 1 follow-up but had insufficient data required for a diagnosis of dementia or MCI at any follow-up visit. Participants in this study ( $\mathrm{n}=$ 2,364 ) had an average age of 75.8 years (standard deviation [SD], 6.4) and average years of school of 10.0 years (SD, 4.8). They were significantly younger and better educated than those excluded because of missing data or lack of follow-up ( $\mathrm{n}=1,329 ; 36 \%$ ), whose average age was 76.6 years (SD, 6.8) and average years of school was 9.2 years (SD, 4.4). The groups did not differ with respect to racial composition, but there were significantly more women

\section{2 cohort 1999 cohort}

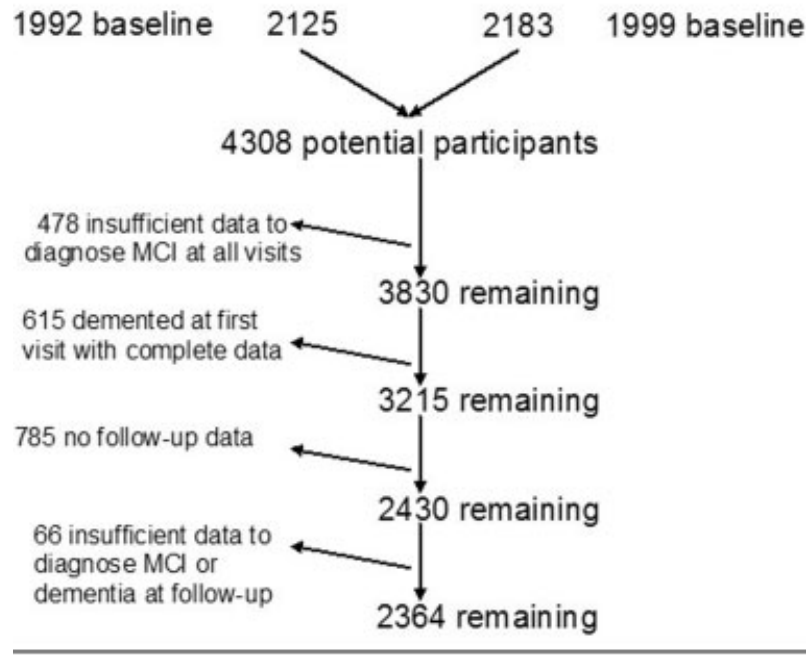

Fig 1. Derivation of the sample for this study from two community-based samples recruited in 1992 and 1999. $M C I=$ mild cognitive impairment. 
$(68.6 \%)$ in the final sample than among those who were excluded (64.9\%). Using a summary measure of medical burden, we found that those who were excluded did not have more medical illnesses than the final sample. As compared with those in the final sample, elders without follow-up were significantly more likely to score less than 1.5 SDs below the demographically matched normative sample on neuropsychological composite scores assessing memory (25 vs 17\%), executive function (21.5 vs $13 \%$ ), visuospatial function $(21.5$ vs $17.4 \%)$, and language function $(23.1$ vs $17.5 \%)$. The average time between follow-up visits in the final sample was 24.3 months (SD, 6.4). Of the sample of 2,364 elders, 823 were reevaluated 1 time, 901 were reevaluated 2 times, 192 were reevaluated 3 times, 174 were reevaluated 4 times, 165 were reevaluated 6 times, 89 were reevaluated 7 times, 9 were reevaluated 8 times, and 11 were reevaluated 9 times.

Of the 2,364 participants in the study, 388 (16.4\%) died at follow-up, and an autopsy was obtained in 27 (7\%) of those who died. As compared with the full sample of 2,337 participants who were not autopsied, the autopsied participants were significantly older at the initial assessment $(76.1 \pm 6.2$ vs $79.3 \pm 6.8$ years; $\mathrm{t}[1,2,362]=2.7 ; p=$ $0.008)$ and had more years of school $(12.5 \pm 4.7$ vs $9.9 \pm$ 4.8 years; $\mathrm{t}[1,2,362]=2.7 ; p=0.007)$ but did not differ with respect to ethnic composition, sex distribution, or proportion of elders who met criteria for MCI.

\section{Assessment Procedures}

\section{RACIAL/ETHNIC GROUP.}

Ethnic group was determined by self-report using the format of the 2000 US Census. ${ }^{22}$ All subjects were first asked to report their race (ie, American Indian/Alaska Native, Asian, Native Hawaiian or other Pacific Islander, black or African American, or white); then in a second question, they were asked whether they were Hispanic.

\section{MEDICAL AND NEUROLOGICAL EVALUATION.}

At the initial visit and each follow-up examination, a physician recorded medical history and medications in a semistructured format. Neurological and physical examinations were performed, including assessment of extrapyramidal signs and functional status.

\section{PSYCHIATRIC STATUS.}

Presence of current depression was determined by asking nine questions that correspond with Diagnostic and Statistical Manual of Mental Disorders, Fourth Edition ${ }^{23}$ criteria for major depressive episode. Current depressive symptoms were assessed using 10 items from the Center for Epidemiological Studies-Depression Scale. ${ }^{24,25}$ Presence of past major depressive episodes, as well as current or past anxiety disorders, psychosis, hallucinations, delusions, psychiatric hospitalizations, psychiatric medications, and alcohol or drug dependence was also assessed. Elderly individuals with depression or other psychiatric disorders were not excluded from the study.

\section{ASSESSMENT OF ACTIVITIES OF DAILY LIVING.}

Items from a Disability and Functional Limitations Scale 26,27 were used to elicit self or observer ratings of instrumental activities of daily living, such as using the telephone, prepar- ing meals, handling money, and completing chores. This instrument has the flexibility to be completed using information from the participant or a collateral, who is a family member, friend, or other person identified by the participant. A summary measure was created, compiling complaints from six domains (using the phone, cooking, shopping, handling finances, making change for purchases, and correctly taking medications). Based on a cutoff capturing $95 \%$ of the normative sample, participants were considered to be functionally intact if they or their caregivers reported difficulty on fewer than three of these items.

\section{MEMORY COMPLAINTS.}

Perceived difficulty with memory was assessed with 11 items from the Disability and Functional Limitations Scale (see earlier) and the Blessed Functional Activities Scale. ${ }^{28}$ Participants were asked whether they had memory difficulties in general, as well as difficulties in specific areas such as memory for names. Participants were considered to have memory complaints if they indicated that they had problems on one or more of the items.

\section{NEUROPSYCHOLOGICAL BATTERY.}

The neuropsychological measures used were selected to assess cognitive functions that are typically affected in dementia and have been shown to effectively distinguish between normal aging and dementia in this community. ${ }^{29}$ The evaluation included measures of learning and memory, orientation, abstract reasoning, language, and visuospatial ability. Specific ability areas and tests administered include verbal list learning and memory (Selective Reminding Test ${ }^{30}$ ), nonverbal memory (multiple-choice version of the Benton Visual Retention Test $[\mathrm{BVRT}]^{31}$ ), orientation (items from the MiniMental State Examination ${ }^{32}$ ), verbal reasoning (Similarities subtest of the Wechsler Adult Intelligence Scale-Revised ${ }^{33}$ ) nonverbal reasoning (Identities and Oddities subtest of the Mattis Dementia Rating Scale $\left.{ }^{34}\right)$, naming (15-item version of the Boston Naming Test ${ }^{35}$ ), letter fluency (Controlled Word Association ${ }^{36}$ ), category fluency (animals, food, and clothing, using procedures from the Boston Diagnostic Aphasia Examination $[\mathrm{BDAE}]^{37}$ ), repetition (high-frequency phrases of the $\mathrm{BDAE}^{37}$ ), auditory comprehension (first six items of the Complex Ideational Material subtest of the $\mathrm{BDAE}^{37}$ ), visuoconstruction (Rosen Drawing $\mathrm{Test}^{38}$ ), and visuoperceptual skills (multiple-choice matching of figures from the BVRT ${ }^{31}$ ). Norms for these tests in this population were developed based on age, years of school, sex, and ethnicity, and were described previously. ${ }^{19}$

\section{CONSENSUS DIAGNOSIS}

After each clinical assessment, a group of physicians and neuropsychologists reviewed the functional, medical, neurological, psychiatric, and neuropsychological data, and reached a consensus regarding the presence or absence of dementia using Diagnostic and Statistical Manual of Mental Disorders, Third Edition Revised criteria. ${ }^{39}$ For follow-up evaluations, this group was shielded from the prior consensus diagnoses. If dementia was diagnosed, the cause was determined using published research criteria for probable and possible $\mathrm{AD},{ }^{40}$ vascular dementia, ${ }^{41}$ Lewy body dementia, ${ }^{42}$ and other de- 
mentias. Severity of dementia was rated using the Clinical Dementia Rating Scale. ${ }^{43}$ Only those who were not diagnosed with dementia were considered for a diagnosis of MCI.

\section{MILD COGNITIVE IMPAIRMENT DIAGNOSTIC CRITERIA.}

MCI criteria were retrospectively applied among nondemented individuals after the consensus conference for each visit. Consistent with standard criteria, ${ }^{2}$ for all subtypes of MCI, those considered for MCI were required to have the following characteristics: (1) a memory complaint (defined earlier); (2) objective impairment in at least one cognitive domain based on the average of the scores on the neuropsychological measures within that domain and a 1.5 SD cutoff using corrections for age, years of education, ethnicity, and sex, and based on the previously established norms; (3) essentially preserved activities of daily living (defined earlier); and (4) no diagnosis of dementia at the consensus conference. A fifth criterion for amnestic MCI is "preserved general cognitive function.” For the MCI subtypes with isolated impairment in one cognitive domain, this criterion was met if neuropsychological test scores in other cognitive domains were not impaired. That is, cognitive criteria for MCIamnestic were met if elders were not impaired on the composite scores for visuospatial, language, and executive function.

To cast the widest net to determine prevalence of MCI and to determine which individuals were more likely to progress to dementia, we expanded the original Petersen criteria, ${ }^{1,2}$ which focus on memory impairment, to include mutually exclusive subtypes based on cognitive features. Our first subtype, MCI-amnestic, corresponded most closely to the original definition that Petersen and colleagues ${ }^{1,2}$ used. Memory impairment was defined as a score less than 1.5 SD below demographically corrected mean on an average composite measure comprising the following learning and memory measures: (1) total recall from the Selective Reminding Test, (2) delayed free recall from the Selective Reminding Test, and (3) recognition from the BVRT. Performance on composite scores from all other cognitive domains (ie, executive, language, and visuospatial) was required to be within reference limits (score had to be $\geq 1.5 \mathrm{SD}$ below the demographically corrected mean). Other MCI subtypes were classified allowing for impairment in a single nonmemory domain if performance on composite scores from all other cognitive domains was within norms. MCI-executive function was assigned if impairment was demonstrated on an average composite measure comprising the following measures: (1) Letter Fluency, (2) Category Fluency, and (3) the Wechsler Adult Intelligence Scale-Revised Similarities subtest. MCI-language was defined as isolated impairment on an average composite measure comprising: (1) Boston Naming Test, (2) BDAE Repetition, and (3) BDAE Comprehension test. MCI-visuospatial was assigned if impairment was demonstrated on an average composite score comprising: (1) Rosen Drawing and (2) BVRT matching. As described in prior studies of MCI (Boyle, 2006 [ref. 9]; Lopez OL, Jagust WJ, DeKosky ST, et al. Prevalence and classification of mild cognitive impairment in the Cardiovascular Health Study Cognition Study: part 1. Arch Neurol 2003;60:1385-1389;
Petersen, RC. Mild cognitive impairment as a diagnostic entity. J Intern Med 2004;256:183-194.), cognition could also be impaired in multiple cognitive domains. MCI-multiple cognitive domains with memory impairment (MCI-MCDM) was diagnosed if there was objective impairment on the memory domain composite score and if there was impairment on at least one other cognitive domain. MCI-multiple cognitive domains without memory impairment (MCIMCDN) was assigned if there was impairment in two or more of the three nonmemory domains and if the memory domain composite score was within norms. Again, classification into the six subtypes was mutually exclusive.

\section{NEUROPATHOLOGY.}

The brains were harvested as soon as possible after death, weighed fresh, and processed according to an upgraded version of a published protocol. ${ }^{44}$ In brief, each brain was divided after a sagittal cut through the corpus callosum. One half was extensively dissected, and blocks were frozen fresh for further investigations. The other half was immersed in buffered, $10 \%$ formalin solution and processed for thorough neuropathological evaluation. For microscopical examination, at least 18 standardized representative blocks were obtained; additional blocks were selected as per the findings on gross examination or documented symptoms. Seven-micrometerthick paraffin sections from all blocks were stained with Luxol fast blue and counterstained with hematoxylin and eosin for general survey. Selected sections were stained with Bielschowsky for evaluation of axons, neuritic plaques, and neurofibrillary and glial tangles; antibodies against $\beta$-amyloid for vascular and parenchymal deposits; phosphorylated tau (AT8) for neuronal and glial tangles; ubiquitin for ubiquitinated cytoplasmic, nuclear, or axonal aggregates; $\alpha$-synuclein for Lewy bodies, Lewy neurites, and glial tangles; or other antibodies as indicated by findings or history. The mean number of neuritic plaques in 5 random $100 \times$ fields per slide was recorded using Bielschowsky-stained slides or $\beta$-amyloid-labeled sections from 7 blocks.

A diagnosis of $\mathrm{AD}$ was assigned according to the criteria of the Consortium to Establish a Registry for Alzheimer's Disease. ${ }^{45}$ The likelihood that dementia was due to the AD changes (neuronal loss, presence of neurofibrillary tangles of $\mathrm{AD}$ and of neuritic plaques) was assessed according to the criteria proposed by The National Institute on Aging, and Reagan Institute Working Group on Diagnostic Criteria for the Neuropathologic Assessment of Alzheimer's Disease. ${ }^{46}$ Furthermore, a Braak and Braak stage was assigned to reflect the extent of involvement of the neurofibrillary tangles of $\mathrm{AD} .{ }^{47} \mathrm{~A}$ brain was assigned to the category of $\mathrm{AD}$ Lewy body variant if there was documented dementia, neuronal loss with neuritic plaques, and neurofibrillary tangles that occurred in numbers of diagnostic significance for $\mathrm{AD}$, and with cortical and subcortical Lewy bodies. The subcortical areas with Lewy bodies included substantia innominata, amygdala, hypothalamus, substantia nigra pars compacta, and nucleus ceruleus. Other causes of dementia were diagnosed according to standard neuropathological criteria available at the time of autopsy. 


\section{Data Analyses}

Demographic and follow-up characteristics of participants who were classified as having MCI were compared with those without MCI using $t$ tests and $\chi^{2}$ analyses. Among those without MCI at baseline, age-specific incidence rates of MCI were calculated within four age groups (65-69, 70-74, $75-79,80+$ years), and 95\% confidence intervals (CIs) about these rates (assuming a Poisson distribution) were calculated separately for the entire population, for men and women, by ethnic group, and by years of education, split at the median ( $0-11$ vs $12+$ years). A Cox proportional hazards model was performed to examine multiple predictors of time to first MCI diagnosis; these predictors were demographic (age, years of education, race/ethnicity, sex), genetic (presence of at least one apolipoprotein $\varepsilon 4$ [APOE- $\varepsilon 4$ ] allele), membership in the 1992 or 1999 cohort, and baseline medical/psychiatric (history of stroke, hypertension, diabetes, heart disease, or psychiatric illness) variables. In these analyses, time to first MCI diagnosis represents the date of progression from normal status to MCI and was calculated as the number of days between the initial neuropsychological evaluation and the neuropsychological evaluation during which MCI was first diagnosed. For those who did not experience development of MCI, time was calculated as the number of days between the initial neuropsychological evaluation and the last neuropsychological evaluation. Agespecific incidence rates of $\mathrm{AD}$ were also calculated among those with and without MCI at baseline. Another Cox proportional hazards model was performed, this time predicting time to $\mathrm{AD}$ diagnosis. In this model, the demographic, genetic, and medical predictors were added as a first set, and the additional contribution of MCI status at baseline was tested by including these variables as a second set of predictors. To determine the individual antecedents of progression to $\mathrm{AD}$, we again used Cox proportional hazards model to predict time to first diagnosis of $\mathrm{AD}$, with demographic (age, education, sex, race/ethnicity, 1992/1999 cohort) and medi$\mathrm{cal} /$ psychiatric (history of hypertension, diabetes, heart disease, stroke, or psychiatric illness) variables entered as a first step, and then allowing individual components of the MCI classification at the initial visit to enter the model using a forward stepwise procedure. We performed univariate analyses of variance (examining any significant differences using Tukey's post hoc tests) and $\chi^{2}$ analyses to compare the characteristics of participants with MCI at baseline who reverted to normal at follow-up versus those who remained classified as MCI or progressed to AD. We also calculated the sensitivity, specificity, and accuracy of MCI or AD using the postmortem diagnosis as the gold standard. The pathological diagnosis of $\mathrm{AD}$ was made if the neuropathologist found sufficient $\mathrm{AD}$ changes in the brain. ${ }^{46,47}$ In some cases, $\mathrm{AD}$ changes were present but were infrequent and did not cross the standard threshold for neuropathological diagnosis of $\mathrm{AD}$. We evaluated the sensitivity and specificity of three clinical states for neuropathological diagnosis of AD: (1) diagnosis of prevalent MCI or incident MCI or incident AD without reversion to normal at follow-up, (2) prevalent or incident impairment in any neuropsychological domain (regardless of AD or MCI diagnosis) without reversion to normal at follow-up, and (3) prevalent or incident neuropsycho- logical impairment in memory (regardless of $\mathrm{AD}$ or $\mathrm{MCI}$ diagnosis) that did not revert to normal at follow-up.

\section{Role of the Funding Source}

The funding source had no role in study design, in the collection, analysis, and interpretation of data, or in the writing of this manuscript. The corresponding authors had full access to all the data in the study and had final responsibility for the decision to submit for publication.

\section{Results}

Sample Characteristics

The mean age of the 2,364 participants was 76.1 years (SD, 6.2 years), and they had an average of 10.0 years (SD, 4.8) of education. The cohort was $28.4 \%$ nonHispanic white, $32.6 \%$ non-Hispanic black, and $39.0 \%$ Hispanic; $68.6 \%$ were women. Only $8 \%$ of the Hispanic subjects were interviewed and tested in English. Information for the Disability and Functional Limitations scale was primarily provided by the participant (97\%). The functional instrument used primarily information from an informant among the remaining $3 \%$ of the sample; this small group of elders was significantly more likely to have significant functional complaints and memory complaints, and was more likely to be classified as having MCI. Informants were present (but may not have been the primary source for the Disability and Functional Limitations instrument) during the initial interview for $6.7 \%$ of the participants, and over the course of all visits, an informant was present for $18 \%$ of the participants. APOE genotype was available for 2,067 subjects $(87 \%)$ in the cohort. At the initial visit, $74.2 \%$ of the participants reported a history of hypertension, 36.3\% heart disease, $23.1 \%$ diabetes, and $8.7 \%$ a history of stroke. An average of 2.3 follow-up evaluations was performed with a mean duration of follow-up of 4.7 years (SD, 2.8). There were no differences in the initial age of participants across the 1992 and 1999 cohorts. Compared with the 1992 cohort, education (in years) was higher in the 1999 cohort (8.6 vs 10.9 years; $\mathrm{t}[2,362]=11.9$; $p<0.001)$. There were more Hispanic subjects in the 1992 cohort (47.3\%) than the 1999 cohort (33.4\%), and fewer non-Hispanic white subjects (20.3\%) in the 1992 cohort compared with the 1999 cohort (33.8\%). Participants in the 1999 cohort were more likely to report stroke $(9.8 \%)$ and heart disease $(39.2 \%)$ than the 1992 cohort (7.1 and 32.1\%, respectively), but the prevalence of hypertension and diabetes was similar. The prevalence of MCI at first visit was greater in the 1992 cohort $(26.9 \%)$ than in the 1999 cohort $(21.8 \%$; $\left.\chi^{2}(1, \mathrm{n}=2,364)=7.9 ; p=0.005\right)$. The length of follow-up was twice as long, on average, among the 1992 cohort (6.7 years) versus the 1999 cohort (3.3 years). 


\begin{tabular}{|ccccc|}
\hline $\begin{array}{l}\text { Table 1. Incident Cases of Mild Cognitive Impairment among Nondemented } \\
\text { Mild Cognitive Impairment at First Visit } \\
\text { MCI Subtype }\end{array}$ & $\begin{array}{c}\text { Incident } \\
\text { MCI } \\
\text { Cases, }\end{array}$ & $\begin{array}{c}\text { Rate per } \\
\text { 100 Person- } \\
\text { years }\end{array}$ & $\begin{array}{c}\mathbf{9 5 \%} \text { CI } \\
\text { (lower) }\end{array}$ & $\begin{array}{c}\mathbf{9 5 \%} \text { CI } \\
\text { (higher) }\end{array}$ \\
MCI with memory impairment & 170 & $2.3 \%$ & $1.9 \%$ & $2.6 \%$ \\
MCI-amnestic & 105 & $1.4 \%$ & $1.1 \%$ & $1.7 \%$ \\
MCD with memory & 65 & $0.9 \%$ & $0.7 \%$ & $1.1 \%$ \\
MCI without memory impairment & 209 & $2.8 \%$ & $2.4 \%$ & $3.2 \%$ \\
MCI-executive & 19 & $0.3 \%$ & $0.1 \%$ & $0.4 \%$ \\
MCI-language & 67 & $0.9 \%$ & $0.7 \%$ & $1.1 \%$ \\
MCI-visuospatial & 85 & $1.1 \%$ & $0.9 \%$ & $1.4 \%$ \\
MCD without memory & 38 & $0.5 \%$ & $0.1 \%$ & $0.7 \%$ \\
All MCI subtypes & 379 & $5.1 \%$ & $4.6 \%$ & $5.6 \%$ \\
MCI = mild cognitive impairment; CI $=$ confidence interval; MCD & $=$ multiple cognitive domains. & \\
\hline
\end{tabular}

Incidence Rates for Mild Cognitive Impairment

Over 7,504.9 person-years of follow-up, there were 379 incident MCI cases (Table 1). Table 2 compares the incidence rates of the 170 cases $(2.3 \% ; 95 \% \mathrm{CI}$, $1.9-2.6 \%)$ in which memory was impaired (MCIamnestic and MCI-MCDM combined) and 209 cases $(2.8 \%$; 95\% CI, 2.4-3.2\%) in which memory was not impaired (MCI-executive, MCI-visuospatial, MCI- language, and MCI-MCDN combined) by age, years of school, race/ethnicity, and sex. The annual incidence rate of MCI-amnestic (1.4\%) was significantly greater than that of MCI-MCDM $(0.87 \%$; incidence rate difference $=0.53 \%$; $95 \%$ CI, $0.19-0.87 \%$ ). A Cox proportional hazards model with time to first diagnosis of MCI (regardless of the subtype) as the outcome demonstrated that as compared with those aged 65 to 69

Table 2. Rate of Progression to Mild Cognitive Impairment (Memory and Nonmemory Impairment Types) per 100 Person-years $(95 \%$ Confidence Interval) according to Age, Education, Ethnicity, and Sex among Elders Who Did Not Have Mild Cognitive Impairment at Baseline $(n=1,800)$

\begin{tabular}{|c|c|c|c|c|c|c|}
\hline Characteristics & $\begin{array}{c}\text { Subjects, } \\
\text { n }\end{array}$ & Person-years & $\begin{array}{c}\text { Incident } \\
\text { MCI-Memory, } \\
\mathbf{n}\end{array}$ & $\begin{array}{l}\text { Rate per } 100 \\
\text { Person-years } \\
(95 \% \mathrm{CI})\end{array}$ & $\begin{array}{c}\text { Incident } \\
\text { MCI- } \\
\text { Nonmemory }\end{array}$ & $\begin{array}{c}\text { Rate per } 100 \\
\text { Person-years } \\
(95 \% \mathrm{CI})\end{array}$ \\
\hline \multicolumn{7}{|l|}{ Age, yr } \\
\hline $65-69$ & 261 & $1,277.3$ & 14 & $1.1 \%(0.5-1.7)$ & 22 & $1.7 \%(1.0-2.4)$ \\
\hline $70-74$ & 588 & $2,588.1$ & 55 & $2.1 \%(1.6-2.7)$ & 67 & $2.6 \%(2.0-3.2)$ \\
\hline $75-79$ & 478 & $1,968.6$ & 44 & $2.2 \%(1.6-2.9)$ & 67 & $3.4 \%(2.6-4.2)$ \\
\hline $80+$ & 473 & $1,674.8$ & 57 & $3.4 \%(2.5-4.3)$ & 53 & $3.2 \%(2.3-4.0)$ \\
\hline \multicolumn{7}{|l|}{ Education, yr } \\
\hline $0-11$ & 1,231 & $3,741.5$ & 94 & $2.5 \%(2.0-3.0)$ & 130 & $3.5 \%(2.9-4.1)$ \\
\hline $12+$ & 1,133 & $3,767.2$ & 76 & $2.0 \%(1.6-2.5)$ & 79 & $2.1 \%(1.6-2.6)$ \\
\hline \multicolumn{7}{|l|}{ Ethnicity } \\
\hline White & 671 & $2,140.6$ & 38 & $1.8 \%(1.2-2.3)$ & 41 & $1.9 \%(1.3-2.5)$ \\
\hline Black & 771 & $2,470.8$ & 57 & $2.3 \%(1.7-2.9)$ & 72 & $2.9 \%(2.3-3.6)$ \\
\hline Hispanic & 922 & $2,897.3$ & 75 & $2.6 \%(2.0-3.2)$ & 96 & $3.3 \%(2.7-4.0)$ \\
\hline \multicolumn{7}{|l|}{ Sex } \\
\hline Male & 743 & $2,253.6$ & 53 & $2.4 \%(1.7-3.0)$ & 62 & $2.8 \%(2.1-3.4)$ \\
\hline Female & 1,621 & $5,255.1$ & 117 & $2.2 \%(1.8-2.6)$ & 147 & $2.8 \%(2.4-3.2)$ \\
\hline
\end{tabular}


years, those aged 70 to 74 years (relative risk [RR], 1.6; 95\% CI, 1.1-2.4), 75 to 79 years (RR, 1.9; 95\% CI, $1.3-2.8$ ), and 80 years and older (RR, 2.5; 95\% CI, 1.7-3.6) were at greater risk for development of MCI. As compared with non-Hispanic white subjects, older adults who self-identified as black (RR, 1.4; 95\% CI, $1.0-1.8$ ) or Hispanic (RR, 1.4; 95\% CI, 1.0-1.9) were also at greater risk for development of MCI. Elders with a history of a diagnosis of hypertension were also at greater risk for development of MCI (RR, 1.4; 95\% CI, 1.1-1.9). In this model, sex, education, cohort, history of heart disease, diabetes stroke, and psychiatric illness were not significant predictors of incident MCI. Among the smaller subsample of 1,572 participants with APOE data and without MCI at the initial visit, older age and hypertension remained risks for incident MCI, but race/ethnicity was no longer a significant predictor of progression to MCI. Presence of the APOE- $\varepsilon 4$ allele was not associated with greater risk for development of MCI. We also performed Cox proportional hazards models with time to first diagnosis of MCI with memory impairment (MCI-amnestic and MCI-MCDM combined) and MCI without memory impairment in the entire sample of people without MCI at the initial visit $(n=1,800)$. Older age and hypertension increased risk for development of MCI with memory impairment, whereas heart disease was protective (RR, 0.70; 95\% CI, 0.50-0.98). Fewer than
12 years of education was the only significant risk factor for development of incident MCI without memory impairment (RR, 1.4; 95\% CI, 1.0-1.9).

\section{Incidence Rate of Alzheimer's Disease and Dementia among Subjects with and without Mild Cognitive Impairment}

Over 10,517.4 person-years of follow-up, there were 309 cases of incident AD. As shown in Table 3, the incidence rates for $\mathrm{AD}$ differed by the presence of MCI. Whereas $10.3 \%$ (186/1,800 subjects) without $\mathrm{MCI}$ at their initial visit were diagnosed with $\mathrm{AD}$ at a follow-up visit, $21.8 \%$ (123/564 cases) with MCI at the first visit were diagnosed with $\mathrm{AD}$ at follow-up. Using a Cox proportional hazards model predicting time to first diagnosis of $\mathrm{AD}$ among the entire sample of 2,364 elders, we found that older participants with fewer than 12 years of school, black and Hispanic subjects, and those with history of diabetes or stroke were at greater risk for development of AD. With all demographic, medical, and psychiatric variables in the model, we found that those with MCI-MCDM, MCIamnestic, MCI-language, and MCI-MCDN initially were more likely to experience development of $\mathrm{AD}$ as compared with elderly individuals without MCI. Table 4 shows RR of developing $\mathrm{AD}$ among MCI subtypes as determined by a Cox proportional hazards model among the subset of 2,067 subjects with APOE geno-

Table 3. Rate of Progression to Alzheimer's Disease per 100 Person-years (95\% Confidence Interval) according to Age, Education, Ethnicity, and Sex among Elderly Subjects with and without Mild Cognitive Impairment at Baseline

\begin{tabular}{|c|c|c|c|c|}
\hline Characteristics & $\begin{array}{c}\text { Total, } \\
\mathbf{n}\end{array}$ & Person-years & $\begin{array}{l}\text { Incident } \\
\text { AD } \\
\text { cases, } \mathbf{n}\end{array}$ & $\begin{array}{l}\text { Rate per } 100 \\
\text { Person-years } \\
(95 \% \mathrm{CI})\end{array}$ \\
\hline No MCI & 1,800 & $8,224.1$ & 186 & $2.3 \%(1.9-2.6)$ \\
\hline Age 65-69 yr & 261 & $1,359.1$ & 3 & $0.2 \%(0.0-0.4)$ \\
\hline Age $70-74$ yr & 588 & $2,921.1$ & 37 & $1.3 \%(0.9-1.7)$ \\
\hline Age $75-79$ yr & 478 & $2,178.6$ & 53 & $2.4 \%(1.8-3.1)$ \\
\hline Age $80+$ yr & 473 & $1,765.3$ & 93 & $5.3 \%(4.2-6.3)$ \\
\hline MCI with memory impairment & 318 & 875.5 & 65 & $7.4 \%(5.7-9.2)$ \\
\hline Age $65-69$ yr & 26 & 126.2 & 4 & $3.2 \%(0.1-6.2)$ \\
\hline Age $70-74$ yr & 73 & 286.6 & 13 & $4.5 \%(2.1-6.9)$ \\
\hline Age $75-79$ yr & 70 & 227.9 & 22 & $9.7 \%(5.8-13.5)$ \\
\hline Age $80+$ yr & 77 & 234.7 & 26 & $11.1 \%(7.1-15.1)$ \\
\hline MCI without memory impairment & 246 & $1,417.9$ & 58 & $4.1 \%(3.1-5.10)$ \\
\hline Age 65-69 yr & 47 & 225.0 & 5 & $2.2 \%(0.3-4.2)$ \\
\hline Age $70-74$ yr & 91 & 417.3 & 12 & $2.9 \%(1.3-4.5)$ \\
\hline Age $75-79$ yr & 90 & 410.6 & 20 & $4.9 \%(2.8-7.0)$ \\
\hline Age $80+y r$ & 90 & 365.0 & 21 & $5.8 \%(3.4-8.1)$ \\
\hline
\end{tabular}




\begin{tabular}{|c|c|c|c|}
\hline \multicolumn{4}{|c|}{$\begin{array}{l}\text { Table 4. Relative Risk of Incident Alzheimer's } \\
\text { Disease Associated with Mild Cognitive Impairment } \\
\text { Status at Baseline }\end{array}$} \\
\hline $\begin{array}{l}\text { Diagnostic } \\
\text { Category }\end{array}$ & $\begin{array}{c}\text { Relative } \\
\text { Risk }\end{array}$ & $\begin{array}{c}\text { 95\% CI } \\
\text { (lower) }\end{array}$ & $\begin{array}{l}\text { 95\% CI } \\
\text { (higher) }\end{array}$ \\
\hline Not MCI & 1 & & \\
\hline MCI-amnestic & 3.2 & 2.1 & 4.7 \\
\hline MCI-executive & 0.7 & 0.2 & 2.8 \\
\hline MCI-language & 2.0 & 1.1 & 3.5 \\
\hline MCI-visuospatial & 1.2 & 0.7 & 2.0 \\
\hline MCI-MCDM & 4.3 & 2.9 & 6.4 \\
\hline MCI-MCDN & 1.9 & 1.1 & 3.0 \\
\hline \multicolumn{4}{|c|}{$\begin{array}{l}\text { Other predictors in the Cox proportional hazards model } \\
\text { predicting time to first diagnosis of Alzheimer's disease (AD) } \\
\text { were age, years of school, sex, race/ethnicity, cohort (1992 vs } \\
\text { 1999), presence of hypertension, diabetes, heart disease, stroke, } \\
\text { and psychiatric history. } \\
\text { MCI = mild cognitive impairment; CI = confidence interval; } \\
\text { MCDM = multiple cognitive domains with memory } \\
\text { impairment; MCDN = multiple cognitive domains without } \\
\text { memory impairment. }\end{array}$} \\
\hline
\end{tabular}

types. These results were essentially identical to that with the entire sample. Not shown in Table 4 is that as compared with those aged 65 to 69 years, those aged 70 to 74 years (RR, 2.6; 95\% CI, $1.4-4.8$ ), 75 to 79 years (RR, 5.0; 95\% CI, 2.7-9.3), and 80 years and older (RR, 11.2; 95\% CI, 6.0-20.1) were at greater risk for development of MCI. Participants with less than 12 years of education were 2.0 times $(95 \% \mathrm{CI}$, 1.4-2.7) more likely to experience development of $\mathrm{AD}$ than those with 12 or more years of education. As compared with non-Hispanic white individuals, older adults who self-identified as black (RR, 2.3; 95\% CI, 1.5-3.5) or Hispanic (RR, 2.4; 95\% CI, 1.6-3.7) were at greater risk for development of $\mathrm{AD}$, as were those with an APOE- $\varepsilon 4$ allele (RR, 1.4; 95\% CI, 1.0-1.7) and history of diabetes (RR, 1.5; 95\% CI, 1.1-1.9) and stroke (RR, 2.3; 95\% CI, 1.6-3.3). Sex, cohort, hypertension, heart disease, and psychiatric history did not significantly influence risk for development of AD. Figure 2 demonstrates the cumulative hazard of development of AD among the MCI subtypes among participants with APOE- $\varepsilon 4$ information. Finally, there were 18 incident cases of non-AD dementia (5 vascular dementia, 2 diffuse Lewy body disease, 2 tumor related, 1 alcohol related, 1 secondary to metabolic dysfunction, 1 secondary to a psychiatric syndrome, and 6 where the cause of dementia could not be determined). There was no change in the results when time to first "all-cause" dementia diagnosis was used as the outcome in these analyses.
Antecedents of Progression to Alzheimer's Disease from Mild Cognitive Impairment

Characteristics of the initial assessment were evaluated as predictors of progression to $\mathrm{AD}$. Adjusting for differences in demographics and medical factors, impairment on the memory composite score was the best predictor of progression to $\mathrm{AD}$ from MCI (RR, 3.00; 95\% CI, 2.32-3.86), followed by isolated impairment in language (RR, 2.09; 95\% CI, 1.61-2.71). Memory complaints entered the model as well (RR, 1.66; $95 \%$ CI, 1.23-2.24), as did isolated impairment in visuospatial function (RR, 1.49; 95\% CI, 1.14-1.94). These results did not change in the smaller sample when APOE- $\varepsilon 4$ status was included in the first set of variables.

\section{Reversal of Mild Cognitive Impairment}

All follow-up visits of the 564 elderly subjects with $\mathrm{MCI}$ at the beginning of the study were examined. We found that $30.2 \%(n=170)$ did not have MCI or dementia at any follow-up visit. About half of those with MCI initially $(\mathrm{n}=264 ; 46.8 \%)$ still had MCI at a subsequent visit and did not revert to normal or progress to dementia.

Individuals with MCI at baseline who progressed to $\mathrm{AD}$ were older, less well educated, more likely to be Hispanic, and reported a history of stroke as compared with both of the groups that did not progress to dementia (Table 5). Those with MCI initially who reverted to normal at follow-up did not differ in age, years of school, sex, race/ethnicity, or presence of medical conditions or an APOE- $\varepsilon 4$ allele from elderly subjects diagnosed with MCI at follow-up. Those without MCI or dementia at any follow-up visit were followed up for less time (3.4 years, an average of 1.7 visits) compared with those with MCI (4.7 years, an average of 2.3 visits) or dementia (5.8 years, an average of 2.9 visits) at follow-up.

To determine whether our results were an artifact of length of follow-up, we limited the analyses to one follow-up only. At the first follow-up of the 564 elderly subjects with MCI at first visit, 45.2\% $(\mathrm{n}=255)$ did not have MCI or dementia, 40.8\% $(\mathrm{n}=230)$ still had MCI, $12.8 \%(\mathrm{n}=72)$ progressed to $\mathrm{AD}$, and $1.2 \%(\mathrm{n}=7)$ progressed to non-AD dementia. Comparisons of background and medical variables among these groups demonstrated that subjects with MCI initially who progressed to $\mathrm{AD}$ at the next visit were older, less well educated, and more likely to report a history of stroke as compared with both of the groups that did not progress, despite having equal years of follow-up. There were no significant differences between the consistent MCI group and the MCI-to-nonMCI group. Of those with MCI who reverted to normal, $21 \%$ no longer met functional complaint criteria, $35 \%$ no longer had memory complaints, and $67 \%$ no 
Table 5. Demographic and Medical Characteristics of Elderly Subjects with Mild Cognitive Impairment at Baseline $(n=564)$ by Diagnostic Outcome, Surveying All Follow-up Visits and Only First Follow-up Visit

\begin{tabular}{|c|c|c|c|c|c|c|}
\hline \multirow[t]{2}{*}{ Characteristics } & \multicolumn{3}{|c|}{ All Follow-up Visits } & \multicolumn{3}{|c|}{ First Follow-up Only } \\
\hline & $\begin{array}{c}\text { MCI to } \\
\text { no } \\
\text { MCI }\end{array}$ & $\begin{array}{c}\text { MCI to } \\
\text { MCI }\end{array}$ & $\begin{array}{c}\text { MCI to } \\
\text { AD }\end{array}$ & $\begin{array}{c}\text { MCI to } \\
\text { no } \\
\text { MCI }\end{array}$ & $\begin{array}{l}\text { MCI to } \\
\text { MCI }\end{array}$ & $\begin{array}{c}\text { MCI to } \\
\text { AD }\end{array}$ \\
\hline $\mathrm{n}$ & 170 & 264 & 123 & 255 & 230 & 72 \\
\hline Mean age, yr & 76.3 & 75.8 & 78.3 & 76.5 & 75.8 & 79.0 \\
\hline Mean education, yr & 9.2 & 9.8 & 6.9 & 8.9 & 9.6 & 7.0 \\
\hline Black, \% & 28.2 & 32.6 & 24.4 & 28.2 & 32.2 & 25.0 \\
\hline Hispanic, \% & 43.5 & 37.9 & 58.5 & 42.0 & 38.0 & 40.1 \\
\hline Women, \% & 68.8 & 68.2 & 66.7 & 71.0 & 63.9 & 70.8 \\
\hline $\begin{array}{l}\text { Apolipoprotein } \varepsilon 4 \\
\text { allele, \% }\end{array}$ & 28.1 & 29.3 & 28.6 & 29.6 & 28.1 & 27.9 \\
\hline Stroke, \% & 8.2 & 6.8 & 12.2 & 6.7 & 7.8 & 16.7 \\
\hline Hypertension, \% & 70.6 & 80.3 & 78.9 & 74.5 & 79.6 & 77.8 \\
\hline Diabetes, \% & 20.6 & 24.6 & 27.6 & 21.6 & 23.9 & 33.3 \\
\hline Heart disease, \% & 31.2 & 35.6 & 41.5 & 34.9 & 33.5 & 44.4 \\
\hline $\begin{array}{l}\text { Average years of } \\
\text { follow-up }\end{array}$ & 3.4 & 4.7 & 5.8 & 2.2 & 2.2 & 2.2 \\
\hline $\begin{array}{l}\text { Average number of } \\
\text { follow-up evaluations }\end{array}$ & 1.7 & 2.3 & 2.9 & 255 & 230 & 72 \\
\hline
\end{tabular}

longer met the neuropsychological criteria at follow-up. There was considerable overlap, such that $48 \%$ reverted because they failed to meet cognitive criteria only, $12 \%$ because of functional criteria only, and $17 \%$

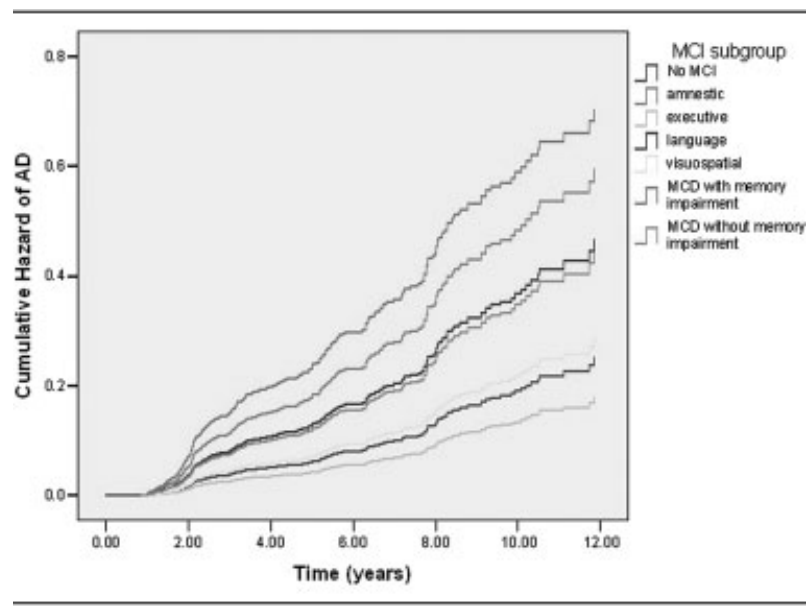

Fig 2. Cumulative hazard of development of Alzheimer's disease $(A D)$ by mild cognitive impairment (MCI) subtype among 2,364 elderly subjects without dementia at initial visit and at least one follow-up visit. Dark blue line indicates no MCI; green line indicates amnestic impairment; gray line indicates executive impairment; purple line indicates language impairment; yellow line indicates visuospatial impairment; red line indicates multiple cognitive domains with memory impairment; light blue line indicates multiple cognitive domains without memory impairment. because of memory complaint criteria only, and the remainder reverted because they failed to meet multiple criteria. The most frequent cause of reversion was no longer meeting cognitive criteria.

We sought to determine whether there were differences in those who reverted to normal within each specific MCI subtype. Surveying all available follow-up visits, elderly subjects with MCI with isolated impairment in one cognitive domain only were the most likely to revert to normal at follow-up $(38.0 \%$ as a group), whereas those with MCI with impairment in multiple cognitive domains were least likely to revert to normal (19.3\%; $\chi^{2}(1[\mathrm{n}=557]=22.2 ; p<0.001$; Table 6) Reversion to normal among those with cognitive impairment in multiple cognitive domains including memory did not differ statistically from the proportion reverting to normal with cognitive impairment in multiple nonmemory domains. There was no difference in the proportion of elders with isolated cognitive impairment in memory, executive function, visuospatial skill, and language reverting to normal at follow-up.

\section{Validity of Antemortem Diagnosis}

Autopsies were obtained in a subsample $(\mathrm{n}=27)$ that was $67 \%$ women, had a mean age of 79.3 years (SD, 6.8) at the initial visit, and a mean education of 12.5 years (SD, 4.7). This subsample was $41 \%$ nonHispanic white, 37\% non-Hispanic black, and 22\% 


\begin{tabular}{|c|c|c|c|c|c|c|}
\hline \multirow[b]{2}{*}{ MCI Subtype } & \multicolumn{2}{|c|}{ MCI to No MCI } & \multicolumn{2}{|c|}{ MCI to MCI } & \multicolumn{2}{|c|}{$\mathrm{MCI}$ to $\mathrm{AD}$} \\
\hline & $\mathbf{n}$ & $\%$ & $\mathbf{n}$ & $\%$ & $\mathbf{n}$ & $\%$ \\
\hline MCI-amnestic & 44 & 35.5 & 51 & 41.1 & 29 & 23.4 \\
\hline MCI-executive & 20 & 44.4 & 23 & 51.1 & 2 & 4.4 \\
\hline MCI-language & 24 & 34.8 & 28 & 40.6 & 17 & 24.6 \\
\hline MCI-visuospatial & 39 & 40.6 & 39 & 40.6 & 18 & 18.8 \\
\hline MCI-MCD with memory impairment & 22 & 18.5 & 61 & 51.3 & 36 & 30.3 \\
\hline MCI-MCD with memory impairment & 21 & 20.2 & 62 & 59.6 & 21 & 20.2 \\
\hline MCI with memory impairment & 66 & 27.2 & 112 & 46.1 & 65 & 26.7 \\
\hline MCI without memory impairment & 104 & 33.1 & 152 & 48.4 & 58 & 18.5 \\
\hline
\end{tabular}

Hispanic. Prevalence of hypertension was 63\%, diabetes was present in $29.6 \%$, and 2 individuals $(7.4 \%)$ reported clinical stroke. The mean interval between last clinical evaluation and death was 24.8 months (SD, 21.3).

Specificity was acceptable (94\%) for neuropathological AD for those diagnosed with prevalent or incident MCI (all subtypes) or incident AD without reversion to normal status, but sensitivity was relatively low $(60 \%)$. Sensitivity was the same $(60 \%)$ when the clinical marker was stable neuropsychological impairment in any cognitive domain (regardless of $\mathrm{MCI}$ or $\mathrm{AD}$ status), but specificity was poor (59\%). Memory complaints had a sensitivity of $70 \%$ for AD pathology but poor specificity $(35 \%)$. Presence of stable neuropsychological impairment in any cognitive domain had perfect specificity $(100 \%)$ for any brain pathology (not limited to AD), but sensitivity was low (62\%). Three of the 27 subjects showed reversion of an MCI diagnosis to normal cognitive status; 2 of these subjects had no detectable abnormality in brain tissue, and 1 had an asymptomatic infarct. False-negative findings included subjects with combined pathology or $\mathrm{AD}$ pathology alone. False-positive findings included primarily infarcts or vascular dementia, but also subjects with lobar atrophy, Parkinson's disease, or brain tissue without recognized abnormality. Among those with AD pathology, time between last clinical evaluation was almost twice as long if clinical diagnosis did not include MCI or $\mathrm{AD}$ (34.7 months; $\mathrm{SD},=31.4$ ) as compared with those with a premortem diagnosis of $\mathrm{AD}$ or MCI (18.8 months; SD, 11.1), but this difference did not reach statistical significance.

\section{Discussion}

This investigation describes the incidence rate of MCI and the progression of $\mathrm{MCI}$ to $\mathrm{AD}$ in a large population-based group of elderly adults from diverse ethnic, linguistic, and educational backgrounds. We found that elder subjects without MCI at the first visit who were older than 70 years and had hypertension were at greater risk for development of MCI. Our AD progression rates among those with $\mathrm{MCI}$ at first visit are comparable with other longitudinal studies of aging in white, non-Hispanic, well-educated participants ${ }^{7,8,11,13,48,49}$ and black individuals in Indiana. ${ }^{14}$ We also found that elders with MCI were at greater risk for development of $\mathrm{AD}$ at follow-up, and that MCI subtypes that included memory impairment are at the greatest risk for $\mathrm{AD}$. We found that participants classified as MCI were not always classified as MCI at follow-up, especially if impairment at first visit was limited to one isolated cognitive domain. Twenty-seven cases underwent autopsy, which demonstrated that antemortem diagnosis of consistent MCI (ie, MCI that did not revert to normal over time) or incident $\mathrm{AD}$ had a $60 \%$ sensitivity and $94 \%$ specificity for $\mathrm{AD}$ at postmortem examination.

We also compared the incidence rates of MCI subtypes. Over an average follow-up of 4.7 years, incidence of amnestic MCI, MCI-visuospatial, and MCIlanguage were highest, whereas incidence of isolated MCI-executive was lowest. There are few epidemiological studies of MCI incidence. Because the mean age of the cohort, the length of follow-up, and the criteria used for MCI differ across studies, ${ }^{11}$ it is difficult to compare incidence rates in this study with other studies. Nevertheless, it appears that our incidence rates of MCI with memory impairment $(2.3 \%$ annual incidence rate) are greater than studies conducted in Germany, ${ }^{50}$ France, ${ }^{49}$ or the United States, ${ }^{11}$ but comparable with the Italian Longitudinal Study on Aging. ${ }^{8}$

Being older than 75 years was the most powerful predictor of progression from $\mathrm{MCI}$ to $\mathrm{AD}$. However, 
MCI status at first visit was also useful in predicting who would go on to experience development of $\mathrm{AD}$. Elderly subjects with MCI-MCDM had the greatest progression rates and were about 4.3 times as likely to develop $\mathrm{AD}$ at follow-up as compared with elderly subjects without MCI. Furthermore, compared with elders without $\mathrm{MCI}$, we found that those with amnestic MCI, MCI-language, and MCI-MCDN were at greater risk for development of $\mathrm{AD}$ at follow-up, but those with MCI-visuospatial and MCI-executive were not. Because progression to $\mathrm{AD}$ was less likely among those with MCI-visuospatial and MCI-executive, this suggests that elders in these categories are less likely to have underlying AD pathology. Therefore, our evaluation of incidence and antecedents of progression to MCI-visuospatial and MCI-executive is less meaningful than the analyses among elders with amnestic MCI, MCI-language, and MCI-MCDN. Evaluation of longitudinal outcomes of different subtypes of MCI can help guide future research to focus on those subtypes that are most likely to progress to $\mathrm{AD}$. It follows that early biomarkers of risk for $A D$ such as plasma $A \beta$, insulin levels, or volume of the hippocampus should show differences from healthy control subjects among elders classified as having amnestic MCI, MCIlanguage, and MCI-MCDN.

We found that medical history of hypertension was associated with MCI, whereas history of diabetes and stroke were risk factors for development of AD. Possession of at least one APOE- $\varepsilon 4$ allele did not predict progression to $\mathrm{MCI}$, and although significant, APOE- $\varepsilon 4$ genotype was a weaker predictor of progression to $\mathrm{AD}$ than $\mathrm{MCI}$ with memory impairment. This finding may relate to the relatively old age of this cohort. Because examination of the relation of these variables to MCI classification was exploratory, further study is needed to determine the cardiovascular and genetic correlates of different MCI subtypes and their interaction with memory function on risk for dementia.

In this study, black and Hispanic subjects, and those with less than a high school education, were not at greater risk for development of $\mathrm{MCI}$; however, these groups were at greater risk for development of $\mathrm{AD}$ even when MCI status at initial visit was taken into account. This finding is likely related to the fact that our neuropsychological criteria for MCI used years of education and norms that were appropriate for the ethnic groups included in the study.

Examination of "reversion to normal" among subjects with MCI was emphasized in this study. If assessed over the course of the entire follow-up period, $30.2 \%$ of our subjects with $\mathrm{MCI}$ at their initial visit were not classified as MCI or demented subsequently. This proportion is comparable with other epidemiological studies, where 14 to $44 \%^{7,11,14}$ of those with MCI at first visit did not have MCI at follow-up. Although we did not find any demographic or medical factors that were associated with instability of diagnosis, we found that MCI with impairment in multiple cognitive domains, with or without memory impairment, was less likely to revert to normal than MCI with impairment in one cognitive domain. This is not surprising because elders with impairment in multiple cognitive domains have poorer overall cognitive function, and thus their neuropsychological test scores are less likely to be on the border of the cutoff. Of those who reverted to normal, most reverted because of cognitive criteria only, with relatively smaller proportions reverting because of functional criteria or memory complaint criteria only. If the cognitive criteria for MCI involve a single "cutoff" of any kind, it is possible that normal variability in cognitive test performance may lead to changes in classification. "Reversions" of this type may not actually represent transitions of one clinical state to another. Continued follow-up of elders who revert to normal should demonstrate whether these individuals are in early stages of dementia (and therefore cognitive function may be expected to show mild fluctuation), whether fluctuation is inherent throughout the course of their follow-up, or whether fluctuation is a marker for "misdiagnosis" of cerebrovascular disease or normal age-related brain changes. We have preliminary support for the latter from our autopsy sample, in which none of the participants who reverted to normal after receiving a diagnosis of $\mathrm{MCI}$ was found to have $\mathrm{AD}$ at postmortem examination.

Compared with other clinicopathological studies, ${ }^{51}$ the sensitivity of antemortem MCI and incident $\mathrm{AD}$ diagnoses to $\mathrm{AD}$ pathology was relatively poor. We suspect this is at least partially explained by the fact that among those with neuropathological $\mathrm{AD}$, the length time between last study visit and death was twice as long in those who were not diagnosed with $\mathrm{AD}$ or MCI clinically as compared with those who were. All of the autopsied elderly cases in our study who were diagnosed with incident $\mathrm{AD}$ were classified as having MCI either at their first visit or a subsequent visit before their AD diagnosis. Specificity was high. Although the autopsy sample was comparable with the overall group with respect to age at baseline, sex, years of education, and ethnic group, a much larger cohort with autopsy is needed before drawing sweeping conclusions about the accuracy of MCI diagnosis with respect to neuropathology.

A limitation of this study is that our average follow-up is only 4.7 years. With longer follow-up, we will be able to compare progression to AD among elderly subjects without MCI at the initial visit who do and do not transition to MCI within the course of the study. Furthermore, it is possible that our conclusions about reversion to normal status after an initial classification as MCI would change with a longer follow-up 
period. It is also possible that those who died before we were able to complete the next follow-up experienced development of MCI or dementia before their death; however, our data do not allow us to estimate the proportion of elderly subjects who may have progressed. To address this problem in future studies, when we discover that a participant has died, we are now administering the Dementia Questionnaire ${ }^{52,53}$ to family members to inquire about cognitive status and functional decline before death.

One other potential limitation is that our cognitive battery did not include traditional measures of attention that would be able to distinguish impairment in this cognitive domain from other domains of cognitive function. It is likely that attentional processes are involved in performance on most, if not all, of the measures that were administered. To the extent that fluency, verbal list learning and recall, and nonverbal memory tap into attentional processes more directly than measures of drawing, perhaps the cognitive domains of language and memory in this study are more "attention loaded" than the visuospatial domain.

The utility of the subjective memory complaint criteria for MCI is controversial because some studies have found that complaints do not improve on the ability to predict progression to $\mathrm{AD} .{ }^{11,12,50} \mathrm{We}$ also found that when the criteria for MCI were allowed to predict progression to $\mathrm{AD}$ separately, neuropsychological impairment in memory conferred a threefold greater risk and impairment in language a twofold greater risk for incident AD. Presence of memory complaints and isolated visuospatial impairment reached statistical significance as a predictor of incident AD, albeit weaker predictors than memory and language impairment. Our autopsy data, although from a limited sample, showed that presence of consistent neuropsychological impairment in one or more cognitive domains was just as good a predictor of $\mathrm{AD}$ pathology as meeting full criteria for $\mathrm{MCI}$ or $\mathrm{AD}$; that is, in this limited sample, presence of a memory complaint did not improve the sensitivity of detection of $\mathrm{AD}$ neuropathology. Specificity of neuropsychological impairment was lower than that of MCI and/or $\mathrm{AD}$, but this might be anticipated given that neuropsychological function may be affected by any brain disorder, not just AD. Specificity of neuropsychological impairment was perfect when the presence of any neuropathology was the gold standard. Furthermore, information from our autopsy sample supports the idea that neuropsychological impairment has better overall accuracy for the presence of $\mathrm{AD}$ pathology than presence of memory complaints, primarily because memory complaints are so prevalent in this age cohort (67\% of our autopsied sample had consistent memory complaints), decreasing their specificity for AD. These clinical and neuropathological results suggest that combining objec- tive neuropsychological test scores and subjective complaint data are useful components of MCI classification, but that perhaps neuropsychological criteria should be given more weight than memory complaints.

This work was supported by National Institute on Aging grants P01-AG07232 (R. Mayeux), P50-AG08702 (R. Mayeux), R01AG16206 (J. Manly), and the Charles S. Robertson Memorial Gift for Alzheimer's Disease Research from the Banbury Fund.

\section{References}

1. Petersen RC, Smith GE, Waring SC, et al. Mild cognitive impairment: clinical characterization and outcome. Arch Neurol 1999;56:303-308.

2. Petersen RC, Doody R, Kurz A, et al. Current concepts in mild cognitive impairment. Arch Neurol 2001;58:1985-1992.

3. Morris JC, Storandt M, Miller JP, et al. Mild cognitive impairment represents early-stage Alzheimer disease. Arch Neurol 2001;58:397-405.

4. Flicker C, Ferris S, Reisberg B. Mild cognitive impairment in the elderly: predictors of dementia. Neurology 1991;41: 1006-1009.

5. Luis CA, Loewenstein DA, Acevedo A, et al. Mild cognitive impairment: directions for future research. Neurology 2003;61: $438-444$.

6. Tabert MH, Manly JJ, Liu X, et al. Neuropsychological prediction of conversion to Alzheimer disease in patients with mild cognitive impairment. Arch Gen Psychiatry 2006;63: 916-924.

7. Ritchie K, Artero S, Touchon J. Classification criteria for mild cognitive impairment: a population-based validation study. Neurology 2001;56:37-42.

8. Larrieu S, Letenneur L, Orgogozo JM, et al. Incidence and outcome of mild cognitive impairment in a population-based prospective cohort. Neurology 2002;59:1594-1599.

9. Boyle PA, Wilson RS, Aggarwal NT, et al. Mild cognitive impairment: risk of Alzheimer disease and rate of cognitive decline. Neurology 2006;67:441-445.

10. Bennett DA, Wilson RS, Schneider JA, et al. Natural history of mild cognitive impairment in older persons. Neurology 2002; 59:198-205.

11. Ganguli M, Dodge HH, Shen C, et al. Mild cognitive impairment, amnestic type: an epidemiologic study. Neurology 2004; 63:115-121.

12. Fisk JD, Merry HR, Rockwood K. Variations in case definition affect prevalence but not outcomes of mild cognitive impairment. Neurology 2003;61:1179-1184.

13. Tschanz JT, Welsh-Bohmer KA, Lyketsos CG, et al. Conversion to dementia from mild cognitive disorder: the Cache County Study. Neurology 2006;67:229-234.

14. Unverzagt FW, Gao S, Baiyewu O, et al. Prevalence of cognitive impairment: data from the Indianapolis Study of Health and Aging. Neurology 2001;57:1655-1662.

15. Manly JJ, Jacobs DM, Sano M, et al. Cognitive test performance among nondemented elderly African Americans and Whites. Neurology 1998;50:1238-1245.

16. Manly JJ, Jacobs DM. Future directions in neuropsychological assessment with African Americans. In: Ferraro FR, ed. Minority and cross-cultural aspects of neuropsychological assessment. Lisse, Netherlands: Swets and Zeitlinger, 2001: 79-96. 
17. Sundquist J, Winkleby MA, Pudaric S. Cardiovascular disease risk factors among older black, Mexican-American, and white women and men: an analysis of NHANES III, 1988-1994. Third National Health and Nutrition Examination Survey. J Am Geriatr Soc 2001;49:109-116.

18. Brancati FL, Kao WHL, Folsom AR, et al. Incident type 2 diabetes mellitus in African American and white adults: the Atherosclerosis Risk in Communities Study. JAMA 2000;283: 2253-2259.

19. Manly JJ, Bell-McGinty S, Tang MX, et al. Implementing diagnostic criteria and estimating frequency of mild cognitive impairment in an urban community. Arch Neurol 2005;62: $1739-1746$.

20. Luchsinger JA, Tang M-X, Shea S, et al. Hyperinsulinemia and risk of Alzheimer disease. Neurology 2004;63:1187-1192.

21. Tang MX, Cross P, Andrews $\mathrm{H}$, et al. Incidence of Alzheimer's disease in African-Americans, Caribbean Hispanics and Caucasians in northern Manhattan. Neurology 2001;56: $49-56$.

22. United States Office of Management and Budget. Revisions to the Standards for the Classification of Federal Data on Race and Ethnicity (October 30, 1997). Standards for maintaining, collecting, and presenting federal data on race and ethnicity. Washington, DC: United States Office of Management and Budget, 1997, 11-6-2000.

23. American Psychiatric Association. Diagnostic and statistical manual of mental disorders. 4th ed. Washington, DC: American Psychiatric Press, 1994.

24. Radloff LL. The CES-D: a self-report depression scale for research in the general population. Appl Psychol Meas 1977;1: $385-401$

25. Kohout FJ, Berkman LF, Evans DA, et al. Two Shorter Forms of the CES-D Depression Symptoms Index. J Aging Health 1993;5:179-193.

26. Golden RR, Teresi JA, Gurland BJ. Development of indicator scales for the Comprehensive Assessment and Referral Evaluation (CARE) interview schedule. J Gerontol 1984;39: $138-146$.

27. Gurland B, Kuriansky J, Sharpe L, et al. The Comprehensive assessment and Referral Evaluation (CARE) - rationale, development and reliability. Int J Aging Hum Dev 1977;8: $9-42$.

28. Blessed G, Tomlinson BE, Roth M. The association between quantitative measures of senile change in the cerebral grey matter of elderly subjects. Br J Psychol 1968;114:797-811.

29. Stern Y, Andrews H, Pittman J, et al. Diagnosis of dementia in a heterogeneous population. Development of a neuropsychological paradigm-based diagnosis of dementia and quantified correction for the effects of education. Arch Neurol 1992;49: 453-460.

30. Buschke H, Fuld PA. Evaluating storage, retention, and retrieval in disordered memory and learning. Neurology 1974;24: 1019-1025.

31. Benton AL. The Visual Retention Test. New York: The Psychological Corporation, 1955.

32. Folstein MF, Folstein SE, McHugh PR. 'Mini-mental State': a practical method for grading the cognitive state of patients for the clinician. J Psychiatr Res 1975;12:189-198.

33. Wechsler D. Wechsler Adult Intelligence Scale-Revised. New York: The Psychological Corporation, 1981.
34. Mattis S. Mental status examination for organic mental syndrome in the elderly patient. In: Bellak L, Karasu TB, eds. Geriatric psychiatry. New York: Grune \& Stratton, 1976: 77-121.

35. Kaplan E, Goodglass H, Weintraub S. Boston Naming Test. Philadelphia: Lea \& Febiger, 1983.

36. Benton AL, Hamsher Kd. Multilingual Aphasia Examination. Iowa City, IA: University of Iowa, 1976.

37. Goodglass H, Kaplan E. The assessment of aphasia and related disorders. 2nd ed. Philadelphia: Lea \& Febiger, 1983.

38. Rosen W. The Rosen Drawing Test. Bronx, NY: Veterans Administration Medical Center, 1981.

39. American Psychiatric Association. Diagnostic and statistical manual of mental disorders. Revised 3rd ed. Washington, DC: American Psychiatric Press, 1987.

40. McKhann G, Drachman D, Folstein M, et al. Clinical diagnosis of Alzheimer's disease: report of the NINCDS-ADRDA Work Group under the auspices of the Department of Health and Human Services Task Force on Alzheimer's disease. Neurology 1984;34:939-944.

41. Roman GC, Tatemichi TK, Erkinjuntti T, et al. Vascular dementia: diagnostic criteria for research studies. Report of the NINDS-AIREN International Workshop. Neurology 1993;43: $250-260$.

42. McKeith IG, Galasko D, Kosaka K, et al. Consensus guidelines for the clinical and pathological diagnosis of dementia with Lewy bodies (DLB): report of the consortium on DLB international workshop. Neurology 1996;47:1113-1124.

43. Morris JC. The Clinical Dementia Rating (CDR): current version and scoring rules. Neurology 1993;43:2412-2414.

44. Vonsattel J-PG, Aizawa H, Ge P, et al. An improved approach to prepare human brains for research. J Neuropath Exp Neurol 1995;54:42-56.

45. Mirra SS, Heyman A, McKeel D, et al. The Consortium to Establish a Registry for Alzheimer's Disease (CERAD). Part II. Standardization of the neuropathologic assessment of Alzheimer's disease. Neurology 1991;41:479-486.

46. The National Institute on Aging, and Regan Institute Working Group on Diagnostic Criteria for the Neuropathological Assessment of Alzheimer's Disease. Consensus recommendations for the postmortem diagnosis of Alzheimer's disease. Neurobiol Aging 1997;18:S1-S2.

47. Braak H, Braak E. Neuropathological stageing of Alzheimerrelated changes. Acta Neuropathol 1991;82:239-259.

48. Panza F, D'Introno A, Colacicco AM, et al. Current epidemiology of mild cognitive impairment and other predementia syndromes. Am J Geriatr Psyhciatr 2005;13:633-644.

49. Solfrizzi V, Panza F, Colacicco AM, et al. Vascular risk factors, incidence of MCI, and rates of progression to dementia. Neurology 2004;63:1882-1891.

50. Busse A, Bischkopf J, Reidel-Heller S, et al. Mild cognitive impairment: prevalence and incidence according to different diagnostic criteria. Br J Psychiatr 2003;182:449-454.

51. Galasko D, Hansen LA, Katzman R, et al. Clinicalneuropathological correlations in Alzheimer's disease and related dementias. Arch Neurol 1994;51:888-895.

52. Kawas C, Segal J, Stewart WF, et al. A validation study of the Dementia Questionnaire. Arch Neurol 1994;51:901-906.

53. Ellis RJ, Jan K, Kawas C, et al. Diagnostic validity of the dementia questionnaire for Alzheimer disease. Arch Neurol 1998; 55:360-365. 